\title{
Las colecciones de hongos en México y su problemática en la biodiversidad del país
}

\author{
GASTĆN GUZMÁN \\ Instituto de Ecología, Apdo. Postal 63, Xalapa, Veracruz, 91000.
}

\begin{abstract}
Resumen. Se presenta un análisis de las colecciones de hongos en México, secas y vivas y se recalca que son pequeñas y poco representativas en comparación con la gran diversidad micológica del país, la cual se estima entre 120,000-140,000 especies. Son 20 los herbarios nacionales que tienen colecciones de hongos, con un total de 220,000 especímenes. Sin embargo en dos de ellos, ENCB y XAL está concentrado más del $50 \%$ de este total. Se hace ver además, que en su mayoría la calidad de dichas colecciones no es buena, porque carece de los datos necesarios para su identificación. Las colecciones de cepas en el país, con no más de 800 unidades, son también escasas y pequeñas en comparación con las del extranjero. Por ejemplo, Gran Bretaña tiene más de 16,500 cepas. Se discute también que la destrucción y/o alteración del medio en México es ya alarmante, lo cual está afectando a los hongos, con graves consecuencias para los ecosistemas.
\end{abstract}

Abstract. An analysis of the Mexican dry and living fungi collections is presented. The collections are poorly represented considering the great mycological diversity of the country, with more than $120,000-140,000$ species. There are 20 national herbaria with fungi collections, with a total of 220,000 specimens. However, two of them, ENCB and XAL have more than the $50 \%$ of this number of specimens. Moreover, the quality of the collections is not satisfactory due to the lack of adecuate data allowing correct specimen identification. The situation of the strain collections in the country is also discussed, with no more than 800 strains; few in comparison with some foreign collections, e.g. Great Briatin has more than 16,500 strains. The problem of the alarming destruction and/or perturbation of the environment in Mexico is presented too, since is already affecting fungi, with serious consequences for ecosystems.

\section{INTRODUCCIÓN}

Los inventarios de plantas y animales y los estudios taxonómicos sobre los mismos, han vuelto a tener relevancia recientemente, luego de que la botánica y la zoología habían sido relegadas a tercer plano porque en apariencia carecían de importancia en nuestro medio. Los trabajos que actualmente se discuten sobre la biodiversidad del planeta, muestran cuan vasta es ésta y sobre todo, lo poco que la conocemos. Por otra parte, en los estudios sobre la biodiversidad se ha ignorado por lo general a los microorganismos (Hawksworth y Ritchie, 1993), a pesar del enorme papel ecológico que tienen estos seres en la naturaleza. En consecuencia, los hongos han quedado descartados, más ahora que se acepta ampliamente que no son plantas sino que forman un grupo independiente de organismos que pertenecen al Reino Fungi, de acuerdo a la propuesta de Whittaker de 1959 acerca de los cinco reinos en que se agrupan los organismos naturales (Herrera y Ulloa, 1990). Es sorprendente saber que los hongos, a pesar de ser el segundo grupo más importante en número de especies en la Tierra, son apenas conocidos (los insectos son el grupo de organismos más grande en el planeta según Wilson, 1992). Hawksworth (1991) calcula que existen más de 1,500,000 especies de hongos en la naturaleza y que de ellos, solamente conocemos apenas el $4.6 \%$. Además, es alarmante descubrir que ese $95.4 \%$ de hongos que falta por conocer, está confinado principalmente en los ecosistemas tropicales, que son precisamente los más afectados y que diariamente se están destruyendo.

En el presente trabajo, se analiza el status de las colecciones de hongos secos y vivos que hay en México, en los herbarios y en los ceparios, respectivamente, con el propósito de hacer una evaluación del escaso desarrollo que ha tenido este renglón en el estudio de la biodiversidad del país, en comparación con la riqueza de la misma.

\section{LA DIVERSIDAD MICOLÓGICA EN MÉXICO Y SU POCO CONOCIMIENTO}

La compleja vegetación que cubre al país, determinada por la gran variación de climas, su intrincada orografía, y a la peculiar posición continental que tiene México entre dos grandes regiones biogeográficas, hace que el territorio nacional cuente con una de las diversidades biológicas más ricas del mundo (Wilson, 1992). Resultado de ello, es el hecho de que los hongos tienen una inmensa diversidad, por encontrar en el país prácticamente todos los hábitats posibles para su desarrollo.

Guzmán $(1994,1995)$ recientemente realizó un análisis sobre cuántos hongos hay en México basándose en los criterios de Hawksworth (1991), de tomar comparativamente el número de especies de otros organismos o el de los hongos de otras latitudes y extrapolarlos a México con todas las reservas y/o consecuencias que ello acarrea. Se estimó que deben de existir en México entre 120,000 y 140,000 especies de hongos. Por otra parte, es altamente significativo saber que se conocen en el país alrededor de 6,000 especies de hongos, por lo que el estado del conocimiento actual sobre la micobiota nacional es de apenas entre el 4.5 y $6 \%$. Además, haciendo una extrapolación con base en el incremento anual del conocimiento de la micobiota nacional de 76 especies por año, se estimó que se necesitarían más de 
1580 años para conocer todos los hongos que crecen en México (!), por lo que es urgente apoyar más las investigaciones sobre la micobiota de México.

\section{LAS COLECCIONES DE HONGOS HERBORIZADOS}

Los hongos en México se empezaron a estudiar desde los inicios de la Colonia en el siglo XVI, pero la primera publicación científica sobre estos organismos fue la de Künth (1822), quien estudió los pocos hongos recolectados por Humboldt y Bonpland en el país, entre 1803 y 1804 . Sin embargo, las colecciones de hongos en el país se iniciaron incipientemente a principios del presente siglo, ya que antes invariablemente, todos los especímenes se depositaban en el extranjero incluso durante las primeras cuatro décadas de este siglo. Guzmán $(1972,1973,1975,1981)$ presentó una reseña sobre los especímenes fúngicos mexicanos en los herbarios del extranjero: BPI, FH, LD, LIL, LSUM, MICH, NO, NY, P y UC, los cuales son no más de 2,000 ejemplares en alrededor de 700 especies. Parece que las primeras colecciones fúngicas en México, fueron las Gándara y A.L. Herrera en el Instituto Médico Nacional, colecciones que después pasaron a integrar las del Instituto de Biología de la UNAM, en donde se formó el Herbario Nacional (MEXU). En un principio, en las décadas de los 30-40's, los hongos de dicho herbario estuvieron a cargo de Ruiz-Oronoz y posteriormente de T. Herrera.

Con referencia a los herbarios nacionales con colecciones de hongos, y revisando los catálogos de Arreguín y Valenzuela (1986) y Arreguín et al. (1994), se encontró que solamente 20 herbarios tienen hongos, con un total de 220,000 especímenes. De éstos, los de ENCB y XAL tiene arriba del $50 \%$, con más de 60,000 y 32,000 especímenes, respectivamente. MEXU, FCME, IBUG, HEIMI e ITCB, en orden de importancia, tienen el $35 \%$ (Guzmán, 1988). Hawksworth y Ritchie (1993) recalcaron que en los países tropicales, solamente el $36 \%$ cuenta con colecciones micológicas y en su relación sobre dichos herbarios al citar a México, hicieron ver que son 12 los herbarios supuestamente más importantes, pero sin anotar ninguna otra cifra.

En el campo de las comparaciones, es interesante observar que Australasia, que representa aproximadamente cuatro veces el territorio nacional, contaba en 1980 con 333,000 especímenes de hongos en más de 10 herbarios (Walker, 1980). Dichas cifras son significativamente más bajas que las de México, en comparación con la magnitud del área australiana. Sin embargo, hay que tomar en cuenta el factor calidad de los especímenes de herbario, que generalmente no se considera en las evaluaciones de los herbarios. La calidad de las colecciones micológicas australianas es superior a la de las mexicanas, por estar respaldando trabajos micológicos publicados por destacados especialistas ingleses o de la región (Cleland, 1934-1935). Desafortunadamente, muchas de las colecciones mexicanas de hongos se encuentran en mal estado, y en la mayoría de ellas los ejemplares no presentan los datos necesarios para su identificación, como son las características en fresco de las fructificaciones. Esto lo ha observado frecuentemente el autor y recientemente Montoya (1994) en el género Lactarius.

Además, los 200,000 especímenes de hongos en los herbarios mexicanos son insuficientes para representar la compleja biodiversidad del país. Tan sólo la colección de hongos del Departamento de Agricultura de E.U.A. (BPI), cuenta con alrededor de un millón de especímenes y el Herbario de Kew en Inglaterra tiene más de 700,000 ejemplares de hongos.

\section{LAS COLECCIONES DE HONGOS VIVOS}

Los ceparios de hongos en México se iniciaron a la par que los estudios sobre levaduras, que se desarrollaron en el Instituto de Biología de la UNAM y en la Escuela Nacional de Ciencias Biológicas del IPN, por los doctores Manuel Ruiz Oronoz y Alfredo Sánchez Marroquín, respectivamente, así como con los estudios de las micoparasitosis por el Dr. Antonio González Ochoa en el Instituto de Salubridad y Enfermedades Tropicales. Esto ocurrió en la década de los 40 's. Posteriormente se formaron otras colecciones en los Laboratorios Syntex, Centro de Estudios Avanzados del IPN, Centro de Postgraduados de Montecillo, Instituto Tecnológico de Ciudad Victoria, INIREB, Instituto de Ecología e Instituto de Micología Neotropical. Estos dos últimos, en Xalapa y Puebla, respectivamente, como consecuencia de la desaparición del INIREB.

No existe ningún catálogo de los ceparios mexicanos. Hawksworth y Richie (1993) presentaron una relación de ocho instituciones nacionales con cepas de microorganismos, con un total de 2,523 cepas, pero no consideraron ni al INIREB ni a las dos instituciones con éste involucradas. La colección más grande que citaron dichos autores, basándose en el catálogo de Gómez-Nava y Contreras-Pérez (1981) es la del Centro de Investigaciones Forestales de Coyoacán con 1016 cepas. Sin embargo, al solicitarle el autor de la presente contribución a la Bióloga Marisela Zamora, Jefe de la Sección de Hongos del aludido centro, que ratificara el número de cepas, se encontró que solamente hay 193 cepas. El cepario del Centro de Estudios Avanzados del IPN cuenta con 212 cepas (Martínez-Cruz, 1982) y el del Instituto de Ecología de Xalapa tiene más de 100 cepas. Del resto de los ceparios en México no hay datos bibliográficos, pero se supone que existen en el país un total aproximado de 800 cepas, tanto de micro como de macromicetos. A manera de comparación, para valorar el desarrollo de los ceparios nacionales, está el trabajo de Hawksworth (1991), quien hizo ver que el $C A B$ International de Gran Bretaña tiene más de 16,500 cepas. A nivel mundial se supone que hay más de 254,000 cepas.

\section{CONSIDERACIONES FINALES}

México es un país con una rica diversidad micológica, con más de 120,000 especies de hongos, debido a su estratégica 
situación biogeográfica y a su compleja orografía. Sin embargo, sus colecciones de hongos son contrastantemente pobres, con apenas 220,000 especímenes, en su mayoría de mala calidad y en 20 herbarios, de los cuales en dos, en ENCB y XAL, se tiene más del $50 \%$ del total. Falta mucho por hacer para conocer y tener representada la micobiota nacional. Se calcula que solamente se conoce el 4.5-6\% de las especies fúngicas del territorio nacional y las monografías sobre géneros o familias de los hongos mexicanos no llegan a diez. Por otra parte, está el alarmante deterioro que se le hace constantemente a la vegetación con graves consecuencias para el hábitat de los hongos y para los ecosistemas en general.

\section{LITERATURA CITADA}

Arreguín M.de L, Valenzuela R. 1986. Segundo catálogo de los herbarios institucionales Mexicanos. Consejo Nacional de la Flora de México, México, D.F.

Arreguín M. de L, Rodríguez-Jiménez C, Fernández-Nava R, Valenzuela R, Méndoza-González AC, Villegas de Gante M. 1994. Historia de los herbarios institucionales y su proyección. Escuela Nacional de Ciencias Biológicas, I.P.N., México.

Cleland JB. 1934-1935. Toadstool and mushrooms and other larger fungi of South Australia. Handbook of the flora and fauna of South Australia, Adelaide. Vols. 1 y 2 (reimpresión James, 1976).

Gómez-Nava M. del S, Contreras-Pérez C.V. 1981. Catálogo de la colección de cultivos de hongos del Instituto Nacional de Investigaciones Forestales. Publ. especial, Instituto Nacional de Investigaciones Forestales, SARH, Coyoacán, México.

Guzmán G. 1972. Macromicetos mexicanos en el Herbario The National Fungus Collections de E.U.A. Bol. Soc. Bot. México 32: 31-55.

Guzmán G. 1973. Hongos mexicanos en los herbarios del extranjero. II. Especies del Herbario Farlow de la Universidad de Harvard, E.U.A. Bol. Soc. Mex. Mic. 7: 121-127.
Guzmán G. 1975. Hongos mexicanos en los herbarios del extranjero. III. Bol. Soc. Mex. Mic. 9: 85-102.

Guzmán G. 1981. Hongos mexicanos en los herbarios del extranjero. IV. Bol. Soc. Mex. Mic. 16: 29-33.

Guzmán G. 1988. Las colecciones de criptógamas en México. En: Memoria del XXX Aniversario del Herbario Nacional Forestal y de la VII Reunión Nacional de Encargados de Herbarios. Publ. especial 62, Instituto Nacional de Investigaciones Forestales y Agropecuarias, SARH, Coyoacán, México.

Guzmán G. 1994. Análisis cualitativo y cuantitativo sobre la diversidad de los hongos en México. En: Halffter, G, edr. La diversidad biológica en Iberoamérica. Vol. 2, Programa Iberoamericano de Ciencia y Tecnología para el Desarrollo, Xalapa (en prensa).

Guzmán G. 1995. La biodiversidad micológica en México. Ciencias, Facultad de Ciencias, UNAM (en prensa).

Hawksworth DL. 1991. The fungal dimension of biodiversity: magnitude, significance, and conservation. Mycological Research 95: 641655.

Hawksworth DL, Ritchie JM. 1993. Biodiversity and biosystematic priorities: microorganisms and invertebrates. $\mathrm{CAB}$ International, Egham.

Herrera T, Ulloa M. 1990. El reino de los hongos. Micología básica y aplicada. UNAM y Fondo de Cultura Económica, México.

Künth CS. 1822. Synopsis plantarum quas in itinere ad plagam aequinoctialem, orbis novi, collegerunt Al. Humboldt et Am. Bonpland. I.F.G. Leurault, París, Vol. I.

Martínez-CruzJ.1982. Catálogo de la colección de cultivos microbianos del Departamento de Biotecnología ý Bioingeniería. Centro de Investigación y Estudios Avanzados, IPN, México, D.F.

Montoya L. 1994. Contribución al conocimiento del género Lactarius (Fungi, Basidiomycotina, Agaricales) en México. Tesis de maestría, Facultad de Ciencias, UNAM, México.

Walker J. 1980. Mycological herbaria and culture collections in Australasia. Department of Agriculture New South Wales, Government Printer, New South Wales.

Wilson EO. 1992. The diversity of life. Norton, Nueva York. 